Revista de Comunicación y Salud, 2018, Vol. 8, n² 2, pp. 1-4

Editado por Cátedra de Comunicación y Salud

ISSN: 2173-1675

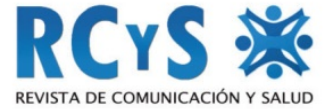

Enviado 17 de mayo de 2018

Aprobado 4 de julio de 2018

\title{
TIPOS DE ANÁLISIS DE COSTOS DE LA ECONOMÍA EN SALUD
}

\author{
Types of cost analysis of the health economy
}

Cristian José Ávila Martínez ${ }^{1}$

Instituto Mexicano del Seguro Social, Zapopan, Jalisco, México.

\section{Resumen}

La importante demanda y aumento en los costos en salud ha generado a nivel mundial y sobre todo en países en vías de desarrollo la generación de evaluaciones económicas para apoyar la toma de decisiones y ofrecer el mayor beneficio al paciente en relación a sus costos. El aumento de la población y la inversión de la pirámide poblacional, así como el aumento del gasto per cápita en salud ha generado que muchos países, sobre todo países en vías de desarrollo, cada vez más invierten en el sector salud, como aquellos que existen en Australia, Canadá e Inglaterra, siendo estos los que a nivel mundial mayor ingreso per cápita invierten.

Palabras clave: costos, economía, salud.

\section{Abstract}

The important demand and increase in the costs in health has generated at global level and especially in developing countries the generation of economic evaluations to support the decision making and to offer the greater benefit to the patient in relation to its costs. The increase in population and investment in the population pyramid, as well as the increase in per capita spending on health, has led to the fact that many countries, especially developing countries, are increasingly investing in the health sector, such as those that exist in Australia, Canada and England, these being the ones that at the highest level per capita income invest.

Keywords: costs, economy, health.

\section{Cómo citar el artículo}

Ávila Martínez, C. J. (2018). Tipos de análisis de costos de la economía en salud. Revista de Comunicación y Salud, 8(2), 1-4.

doi: http://doi.org/10.35669/revistadecomunicacionysalud.2018.8(2).1-4

\footnotetext{
${ }^{1}$ Autor para correspondencia: Cristian José Ávila Martínez dr.cristian martinez@hotmail.com
} 
Tipos de análisis de costos de la economía en salud

\section{OBJETIVO}

Conocer los diferentes tipos de análisis de costos dentro de la economía en salud, así como saber cuáles son sus diferencias y formas de implementación, su importancia y beneficio en pro a la toma de decisiones y realizaciones de estrategias gubernamentales para contribuir en el desarrollo del país dentro del sector salud.

\section{INTRODUCCIÓN}

México es la undécima mayor economía del mundo, la cual ha pasado de una economía dependiente del petróleo a principios de la década de 1990 a un centro manufacturero después del tratado de libre comercio, pero se encuentra en el último lugar en cuanto al gasto en salud, si bien los primeros rubros para dividir las riquezas son la infraestructura, capacitación, salud y reducción de la pobreza, '1buscándose siempre que los recursos se dividan de manera eficiente aumentando la producción y con el menor coste posible ${ }^{2}$.

El ingreso continúa sumamente concentrado en pocos, mientras que bastantes familias aún viven en la pobreza, la inseguridad es alta, continua el desaprovechamiento escolar y aun en nuestro país existe discriminación hacia la mujer. Lamentablemente hoy en día el crecimiento no ha sido suficientemente incluyente para lograr mejores condiciones de vida para muchas familias mexicanas y en lo que respecta a la salud se han puesto en marcha diferentes estrategias e iniciativas para mejorar la salud como lo es: Progresa en 1997, oportunidades en 2002 y prospera creado en 2014, así como actualmente funciona el sistema PREVENIMS en el Instituto Mexicano del Seguro Social ${ }^{1}$.

La evaluación económica la podemos definir como el análisis comparativo de las acciones alternativas en pro a la salud, siendo el análisis de costos la parte central de la evaluación, análisis que se debe de realizar frente al inminente crecimiento de procedimientos diagnósticos y terapéuticos, mientras que los costos son todos aquellos que se reducen en un gasto económico, debiéndose recalcar que los costos relevantes a considerar dependen mucho de la perspectiva que asume la evaluación, pues los pacientes, prestadores y financistas, tienen perspectivas distintas, porque trasladan costos de forma diferente ${ }^{3}$, de ahí entonces las desigualdades en ocasiones por desabasto de cierto medicamento en primer nivel y la introducción de un nuevo fármaco en su lugar, el establecer cierta terapia en vez de alguna otra, siempre teniéndose en balanza el menor costo con el mayor beneficio, este último siendo relativo por las autoridades quienes lo deciden, que no muchas veces pertenecen al ámbito médico, sino solo al económico.

Existen cuatro tipos principales de análisis de costos en la economía de la salud: análisis de costo-minimización, análisis costo-efectividad, análisis costo-utilidad y análisis costo-beneficio. Si bien todos estos análisis emplean una metodología similar en la estimación de costos, se diferencian en el método utilizado para estimar los beneficios, pues lo que tienen en común es la reducción de costos con el mayor beneficio ${ }^{4}$.

Revista de Comunicación y Salud, 2018, Vol. 8, n² 2, pp. 1-4 
Los análisis de costo-minimización son aquellos que comparan exclusivamente los costos de dos intervenciones alternativas bajo el supuesto que ambas proveen un nivel de beneficio equivalente, existen pocos estudios de este tipo debido a su dificultad en que dos intervenciones provean exactamente los mismos beneficios ${ }^{4}$.

Los análisis de costo-efectividad son de tipo unidimensional, pues los beneficios de las estrategias a evaluar no son equivalentes y son medidos en unidades naturales de morbilidad, mortalidad o calidad de vida, como por ejemplo: muertes evitadas, los años de vida ganados, cambios en unidades de presión arterial o colesterol ${ }^{4}$. Comparan para cada tratamiento o prueba diagnóstica los costos médicos que implica el manejo de la enfermedad, frente al beneficio clínico ${ }^{5}$.

El inconveniente de este tipo de estudio es que no se pueden comparar tratamientos cuya efectividad se mide de forma distinta ${ }^{6}$.

Dentro de los análisis de costo-beneficio se requieren que las consecuencias de la intervención a evaluar sean expresadas en términos monetarios, lo que permite al analista hacer comparaciones directas entre distintas alternativas por medio de la ganancia monetaria neta o razón de costo-beneficio. El principal inconveniente de los análisis costo-beneficio está en la dificultad de asignar un valor monetario al efecto terapéutico deseado y el desconocimiento de los efectos secundarios del medicamento ${ }^{7}$.

El análisis costo-utilidad se basa en la valoración de las preferencias o utilidades de los individuos y la satisfacción en relación a la calidad de vida, expresando el resultado en términos de calidad de años de vida. Aunque el costo de un medicamento o procedimiento debe ser parte de la decisión, no debe ser el único criterio para el uso del medicamento, las preferencias del paciente también deben ser consideradas, esto último valorado como la utilidad la cual se mide del 0 al 1 , donde el 0 corresponde a la muerte y el 1 la mejor salud posible. El inconveniente que se tiene respeto a este tipo de análisis de costos es que la opinión del paciente sano o enfermo puede varias dependiendo a su perspectiva e intereses personales, modificándose los resultados drásticamente cunado se hacen en diferentes tipos poblacionales ${ }^{8}$.

\section{CONCLUSIÓN}

La repartición de las riquezas siempre ha sido un tema muy importante para el sector salud, en el cual si existe una mala administración o decisiones inadecuadas prevalecerá la carencia en la atención a la salud y sobre todo en la población de difícil acceso como lo es aquellas que viven en la pobreza como la que se vive en México, para ello han existido desde los países de primer mundo como Inglaterra agencias gubernamentales que se encargan de atender las demandas a la salud mediantes análisis e costos económicos en pro del beneficio para su población; ya sea utilizándose cualquiera de los cuatro tipos de estudios de costos, como lo es minimización de costos, costo-beneficio, costo-utilidad o costo-efectividad todos ellos conllevan a un mismo fin dependiendo los intereses de la organización que los realice y de aquellos miembros que lo hagan, ya que no todo el tiempo son médicos quienes 
implementan estas acciones sino economistas, políticos o aquellos donde solo se observa el valor monetario y no la salud propiamente dicha. El crecimiento del gasto sanitario público y la demanda generalizada de las instituciones sanitarias los coloca lejos de prestar sus servicios con la eficiencia deseable por lo que se exige un cambio en la concepción de la información de costos, ya que dependerá de la perspectiva de quien realice el análisis la mejoría de la salud o economía del país, el análisis de costos ofrece grandes posibilidades a la gestión hospitalaria, llegando a convertirse en una importante herramienta para la toma de decisiones, pero siempre en balance entre estos dos polos lo económico y la salud, con tendencia a la elección del mejor beneficio al menor costo, con la intención de abastecer la oferta de la gran demanda que existe como lo es en nuestro país México.

\section{BIBLIOGRAFÍA}

Comité de Análisis Económico y del Desarrollo (2017). Estudios económicos de la OCDE, Mejorespolíticas para una vida mejor.

Hernández, P.; Arredondo A.; Ortiz C. y Rosenthal G. (1995). Avances y retos de la economía en salud. Rev. salud pública, 29(4), p. 326-332.

Lenz, R. (2010). Análisis de costos en evaluaciones económicas en salud: Aspectos introductorios. Rev. Med. Chile, 138(2), p. 88-92.

Zarate V. (2010). Evaluaciones económicas en salud: Conceptos básicos y clasificación. Rev. Med. Chile, 138(2), p. 93-97.

Buendía J. A. (2014). Aspectos básicos para la interpretación. Revista americana de medicina respiratoria, 14(2), p. 107-110.

Pinto J. L.; Puig J. y Ortún-Rubio, V. (2001). Análisis coste-utilidad. Atención Primaria, 27(8), p. 569-573.

Santamaría, A. M.; Herrera, J. E.; Sil P. A., et al. (2015). Estructura, sistemas y análisis de costos de la atención. Medicina e investigación, 3(2), p. 134-140.

Sociedad Colombiana de Anestesiología y Reanimación (2002). Tipos de análisis de costos: Organización científico- gremial. Curso de fármacoeconomía. Unidad 2. 